\title{
Outcome of treatment with EMA/EP (etoposide methotrexate and actinomycin-D/ etoposide and cisplatin) regimen in gestational trophoblastic neoplasia
}

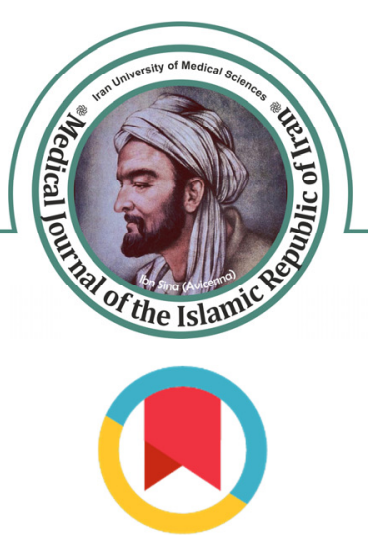

\author{
Soheila Aminimoghaddam*1, Forough Nezhadisalami ${ }^{1}$, Shabnam Anjidani ${ }^{2}$, Saeedeh Barzin Tond ${ }^{1}$
}

Received: 22 Nov 2017

Published: 3 May 2018

\section{Abstract}

Background: Gestational trophoblastic neoplasia (GTN) originates from placental trophoblast and is a highly chemosensitive and curable gynecologic malignancy. The present study was conducted to evaluate the effectiveness and safety of EMA/EP (etoposide, methotrexate, actinomycin-D, etoposide, and cisplatin) regimen in the treatment of high-risk GTN as well as patients' outcome.

Methods: Hospital charts of all patients with confirmed diagnosis of high-risk GTN who received EMA/EP regimen treatment during a 12-year period (2001-2012) at the tertiary center of comprehensive women's hospital in Tehran, Iran, were reviewed from 2012 to 2013, retrospectively.

Results: In this study, 25 patients with GTN who were treated with EMA/EP regimen during the study were identified. Complete remission rate in GTN patients with failure of single agent chemotherapy who were treated with EMA/EP regimen, as the first- line treatment, was $100 \%$, while it was $81 \%$ in those with primary high-risk GTN. Overall remission rate in high-risk GTN patients treated with EMA/EP regimen was $88 \%$. Anemia (92\%) and leucopenia (72\%) were the most common adverse effects of EMA/EP chemotherapy regimen. Acute myeloid leukemia (AML) and mortality, as the most severe adverse effects of EMA/EP regimen, were seen only in 1 patient.

Conclusion: According to the results, EMA/EP regimen could induce complete remission in $88 \%$ of patients with high-risk GTN. Application of EMA/EP is recommended as the first- line therapy in patients with failure of single agent chemotherapy. However, proper care should be considered to prevent and reduce EMA/EP hematologic toxicity.

Keywords: EMA/EP regimen, Chemotherapy, Gestational trophoblastic neoplasia

Copyright $\odot$ Iran University of Medical Sciences

Cite this article as: Aminimoghaddam S, Nezhadisalami F, Anjidani Sh, Barzin Tond S. Outcome of treatment with EMA/EP (etoposide methotrexate and actinomycin-D/ etoposide and cisplatin) regimen in gestational trophoblastic neoplasia pproach. Med J Islam Repub Iran. 2018(3 May);32:36. https://doi.org/10.14196/mjiri.32.36

\section{Introduction}

Gestational trophoblastic neoplasia (GTNs) are malignant tumors that originate from placental trophoblast (1). They usually occur following an antecedent pregnancy that may be a term pregnancy, miscarriage, and an ectopic or a molar pregnancy. GTNs often develop following a molar pregnancy (2). The risk of developing GTN following complete hydatidiform mole is approximately $15 \%$, while it is $0.5 \%$ to $1 \%$ after partial hydatidiform mole (3).

The incidence of GTN is different in different geograph-

Corresponding author:Dr Soheila Aminimoghaddam,aminimoghaddam.s@iums.ac.ir 1. Department of Gynecology and Oncology, Iran University of Medical Sciences, Tehran, Iran.

2. Iran University of Medical Sciences, Tehran, Iran. ical regions, and it is higher in Asia than in Western countries (4). No accurate data exist on the incidence of GTN in Iran, but its incidence in Turkey has been reported to be $1 / 1000$ deliveries.

GTN is a curable gynecologic malignancy, which is highly sensitive to chemotherapy (5), and its cure rate has increased to $98 \%$ by effective treatment (2). Currently, the complete cure rate of about $100 \%$ is predictable for nonmetastatic and low-risk GTN, while preserving the patient's fertility $(6,7)$. However, the cure rate ranges from

\section{$\uparrow$ What is "already known" in this topic:}

EMA-CO is recommended as the regimen of choice for initial treatment of high-risk GTN. However, $25 \%$ to $30 \%$ of patients with high-risk GTN still need to undergo salvage chemotherapy. EMA-EP is one of the alternative regimens with a survival rate of up to $84.9 \%$.

$\rightarrow$ What this article adds:

This study revealed that EMA/EP regimen as the first line treatment could induce complete remission in $88 \%$ of patients with high-risk GTN. 
$80 \%$ to $90 \%$ in high-risk GTN (8).

Low-risk GTNs are usually treated with single agent chemotherapy, while high-risk GTNs should be treated with chemotherapy. However, about $25 \%$ to $30 \%$ of GTN tumors are resistant to therapy.

Various agents have been used for combination chemotherapy of high-risk GTN. EMA/CO (etoposide, methotrexate, actinomycin D, cyclophosphamide and oncovin $($ /vincristine) is the most commonly administered first-line combination regimen for high-risk $\operatorname{GTN}(9,10)$. EMA/CO regimen is relatively well-tolerated and has a good cure rate, however, $25 \%$ to $30 \%$ of patients with highrisk GTN still need further salvage chemotherapy $(2,5)$, and the results are not satisfactory in metastatic cases (9). Due to the limitations of combination chemotherapy, it is necessary to find an alternative regimen for patients who have failed to respond to available combination agents; also, it is highly important to look for alternative chemotherapeutic regimen without such deficiencies as the firstline treatment.

One of the alternative regimens for EMA/CO is EMA/EP (etoposide, methotrexate, actinomycin $\mathrm{D} /$ etoposide, cisplatin) in which cyclophosphamide and vincristine in the EMA/CO are replaced with etoposide and cisplatin.

Due to the higher response rate of EMA/EP, compared to EMA/CO, some authors have suggested EMA/EP as the first- line chemotherapy regimen (11). EMA/EP has been used as a good salvage treatment with a survival rate of up to $84.9 \%(8)$. As the first-line treatment, EMA/EP has been reported to have survival rate of $88 \%$ in high-risk GTN (12). However, limited data are available about the efficacy and toxicity of EMA/EP regimen in the treatment of highrisk GTN.

The present study was conducted to investigate the efficacy and safety of EMA/EP regimen in the treatment of high-risk GTN as well as the patients' outcomes.

\section{Methods}

This was a retrospective review of hospital records of all patients with high-risk GTN treated with EMA/EP regimen as the first-line treatment during a 12-year period (20012012) at the tertiary center of comprehensive women's hospital in Tehran, Iran.

The study protocol was approved by the Institutional Review Board (IRB) of Iran University of Medical Sciences (ethical number: IR.IUMS.FMD.REC. 1392.801011133).

Hospital records of all patients who referred to the gynecology-oncology ward of our hospital from 2001 to 2012 were screened. Demographic data and gynecological and obstetrical history, such as gravidity, parity, abortion, and treatment side effects, were recorded using questionnaires. Anatomical and clinical staging system was performed following the confirmation of persistent GTN with rising or plateau of serum $\beta$-HCG concentration. When the disease was confined to the uterus, stage I or nonmetastatic form was perceived; on the other hand, when a metastatic lesion was detected in the vagina, lung, etc., stage II, III, or IV were considered. Disease scoring was done according to WHO (World Health Organization) scoring system, which included age, duration of the disease, prior pregnancy type, serum $\beta$-HCG titer, size and number of metastasis. Patients with the risk score of 7 or greater were categorized as having high-risk GTN and those who obtained the score of 6 or less as having low- risk GTN.

Two groups of patients were included in the study who received EMA/EP regiment:

(1) Patients with risk score of 7 or greater based on modified WHO and FIGO (International Federation of Gynecology and Obstetrics) scoring system, and (2) those whose initial single agent chemotherapy failed (high-risk GTN) and had GOG (Gynecologic Oncology Group) performance status of less than 3 with normal primary $\mathrm{CBC}$ ) complete blood count), liver, and kidney test.

Patients with chronic liver or kidney disease or other diagnoses were excluded.

In this study, 25 patients with high-risk GTN who were treated with EMA/EP regimen were identified during April 2001 and April 2012.

To evaluate response to treatment, serum $\beta$-HCG was measured every 2 weeks using radioimmunoassay method,

Table 1. EMA/EP chemotherapy regimen in patients with high-risk GTN with and without brain and liver metastasis

\begin{tabular}{|c|c|c|}
\hline Time & Regimen & Protocol \\
\hline \multicolumn{3}{|c|}{ For high-risk GTN without brain metastasis } \\
\hline First day & Etoposide (vp-16) & $100 \mathrm{mg} / \mathrm{m}^{2}$ in $500 \mathrm{ml}$ normal saline by IV infusion over $30 \mathrm{~min}$ \\
\hline First day & Actinomycin & $0.5 \mathrm{mg}$ IV stat \\
\hline First day & Methotrexate & $100 \mathrm{mg} / \mathrm{m}^{2} \mathrm{IV}$ stat \\
\hline First day & Methotrexate & $200 \mathrm{mg} / \mathrm{m}^{2}$ in $500-1000 \mathrm{ml}$ normal saline by IV infusion over 12 hours \\
\hline Second day & Etoposide (vp-16) & $100 \mathrm{mg} / \mathrm{m}^{2}$ in $500 \mathrm{ml}$ normal saline by IV infusion over $30 \mathrm{~min}$ \\
\hline Second day & Actinomycin_D & $0.5 \mathrm{mg}$ IV stat \\
\hline Second day & Citrovorum factor rescue & $15 \mathrm{mg} \mathrm{IM} / \mathrm{PO}$ every 12 hours, 4 doses to start 24 hours commencement of methotrexate \\
\hline Eighth day & Etoposide (vp-16) & $100 \mathrm{mg} / \mathrm{m}^{2}$ in $500 \mathrm{ml}$ normal saline by IV infusion over $30 \mathrm{~min}$ \\
\hline Eighth day & Cisplatin & $60-80 \mathrm{mg} / \mathrm{m}^{2} \mathrm{IV}$ \\
\hline \multicolumn{3}{|c|}{ For high-risk GTN with brain metastasis } \\
\hline First day & Etoposide (vp-16) & $100 \mathrm{mg} / \mathrm{m}^{2}$ in $500 \mathrm{ml}$ normal saline by IV infusion over $30 \mathrm{~min}$ \\
\hline First day & ActinomycinD & $0.5 \mathrm{mg}$ IV stat \\
\hline First day & Methotrexate & $100 \mathrm{mg} / \mathrm{m}^{2} \mathrm{IV}$ stat \\
\hline First day & Methotrexate & $1000 \mathrm{mg} / \mathrm{m}^{2}$ in $500-1000 \mathrm{ml}$ normal saline by IV infusion over 12 hours \\
\hline Second day & Etoposide (vp-16) & $100 \mathrm{mg} / \mathrm{m}^{2}$ in $500 \mathrm{ml}$ normal saline by IV infusion over $30 \mathrm{~min}$ \\
\hline Second day & ActinomycinD & $0.5 \mathrm{mg}$ IV stat \\
\hline Second day & Citrovorum factor rescue & $30 \mathrm{mg} \mathrm{IM} / \mathrm{PO}$ every 12 hours, 6 doses to start 24 hours commencement of methotrexate \\
\hline Eighth day & Etoposide (vp-16) & $100 \mathrm{mg} / \mathrm{m}^{2}$ in $500 \mathrm{ml}$ normal saline by IV infusion over $30 \mathrm{~min}$ \\
\hline Eighth day & Cisplatinum & $60-80 \mathrm{mg} / \mathrm{m}^{2}$ in $100 \mathrm{ml}$ normal saline by IV infusion (not to exceed $1 \mathrm{mg} / \mathrm{min}$ ) \\
\hline Repeated col & days 15,16 , and 22 & \\
\hline
\end{tabular}


and a serum $\beta$-HCG level $<5$ IU/L (normal level) for 3 consecutive weeks was considered as positive response to the treatment or remission.

EMA/EP chemotherapy was started (Table 1) for the patients and continued until serum $\beta$-HCG levels reached the normal level $(<5 \mathrm{IU} / \mathrm{L})$. Then, 1 to 3 additional courses of consolidation chemotherapy were administered for 6 weeks. Chemotherapy courses were repeated every 2 weeks during the course of treatment.

The remission rate of the disease was evaluated by $\beta$ HCG level and documented in patients' medical records. Moreover, the safety and efficacy of the treatment were measured according to NCI Common Terminology Criteria for Adverse Events (CTCAE). Patients with confirmed brain metastasis received high-dose regimen (Table 1). They also received concurrent radiotherapy of $3000 \mathrm{cGy}$ to the whole brain.

All patients received GCSF (granulocyte colony-stimulating factor) 24 hours after completion of EMA/EP chemotherapy course and continued taking it up to 24 hours before the next course of chemotherapy to prevent or reduce hematologic complications of the treatment.

Chemotherapy tolerance, remission, recurrence, and toxicity were monitored during the treatment period. Moreover, recurrence, treatment complications, and toxicity were recorded after remission.

\section{Statistical analysis}

Data were analyzed using SPSS software version 17.0 for Windows. Continuous data were expressed as mean \pm SD and categorical data as number and percent. P- value less than 0.05 was considered as statistically significant.

\section{Results}

The mean age of the patients was $30.0 \pm 6.1$ years (range: 18-44). Demographic characteristics of the patients are demonsrtated in Table 2. Most GTN patients (68\%) were in the stage III of the disease with lung metastasis based on FIGO anatomical staging. Moreover, most of GTN tumors occurred following molar pregnancy (48\%) (Table 2). Precedent pregnancies were terminated by D\&C (dilatation and curettage) in $76 \%$ (19) of patients, vaginal delivery in $20 \%$ (5), and salpingectomy in 4\% (1). Type of GTN pathology was unknown in most patients (Table 2). Uterine mass was detected in 16 of 20 patients $(84 \%)$, and tumor size was less the $5 \mathrm{~cm}$ in $52 \%$ of the cases (11). Four patients underwent hysterectomy, of which 3 had the tumor size of greater than $5 \mathrm{~cm}$.

A total of 78 courses of EMA/EP chemotherapy and 44 consolidation courses were given (Table 3). The main therapeutic outcome was remission rate. Remission rate in the metastatic group was $88 \%$ and it was $100 \%$ in the nonmetastatic group (Table 3). After failure of monotherapy, total chemotherapy courses were less in patients (the second group of patients) than those with primary high-risk GTN (the first group of included patients) (Table 3).

The most common toxicity of EMA/EP regimen was myelosuppression, including anemia and leucopenia, which were observed in $92 \%$ and $72 \%$ of the patients, respectively (Table 4).

Table 2. Demographic characteristics of patients with high-risk GTN

\begin{tabular}{|c|c|c|}
\hline Variable & & $\begin{array}{c}\begin{array}{c}\text { Value } \\
(\mathrm{n}=25)\end{array}\end{array}$ \\
\hline \multirow[t]{2}{*}{ Gravid } & $1-4$ & $20(80)$ \\
\hline & $5-8$ & $5(20)$ \\
\hline \multirow[t]{2}{*}{ Para } & Yes & $22(88)$ \\
\hline & No & $3(12)$ \\
\hline \multirow[t]{2}{*}{ Abortion } & Yes & $11(44)$ \\
\hline & No & $14(56)$ \\
\hline \multirow[t]{2}{*}{ Live birth } & Yes & 21(84) \\
\hline & No & $4(16)$ \\
\hline \multirow[t]{2}{*}{ Mole } & Yes & $16(64)$ \\
\hline & No & $9(36)$ \\
\hline \multicolumn{3}{|l|}{ FIGO anatomical staging } \\
\hline \multicolumn{2}{|l|}{ Stage 1 . disease confined to the uterus } & $5(20)$ \\
\hline \multicolumn{2}{|l|}{ Stage 2. GTN extends to the genital structures } & $0(0)$ \\
\hline \multicolumn{2}{|l|}{ Stage 3 . lung metastasis } & $17(68)$ \\
\hline \multicolumn{2}{|l|}{ Stage 4. liver and/or brain metastasis } & $3(12)$ \\
\hline \multicolumn{3}{|l|}{ Indication for treatment } \\
\hline \multicolumn{2}{|l|}{ Failure of single-agent therapy } & $9(36)$ \\
\hline \multicolumn{2}{|l|}{ Primary high-risk GTN } & $16(64)$ \\
\hline \multicolumn{3}{|l|}{ Antecedent pregnancy } \\
\hline \multicolumn{2}{|l|}{ Hydatiform mole } & $12(48)$ \\
\hline \multicolumn{2}{|l|}{ Term pregnancy } & $8(32)$ \\
\hline \multicolumn{2}{|l|}{ Non-molar abortion } & $4(16)$ \\
\hline \multicolumn{2}{|l|}{ Ectopic pregnancy } & $1(4)$ \\
\hline \multicolumn{3}{|l|}{ Pathology } \\
\hline \multicolumn{2}{|l|}{ Choriocarcinoma } & $8(3)$ \\
\hline \multicolumn{2}{|l|}{ Extrauterine choriocarcinoma, } & $1(4)$ \\
\hline \multicolumn{2}{|l|}{ Unknown } & $16(64)$ \\
\hline \multicolumn{3}{|l|}{ Therapeutic Surgery for GTN } \\
\hline \multicolumn{2}{|l|}{ Hysterectomy } & $4(16)$ \\
\hline \multicolumn{2}{|l|}{ Nephrectomy } & $1(4)$ \\
\hline \multicolumn{2}{|l|}{ Thoracotomy } & $2(16)$ \\
\hline
\end{tabular}




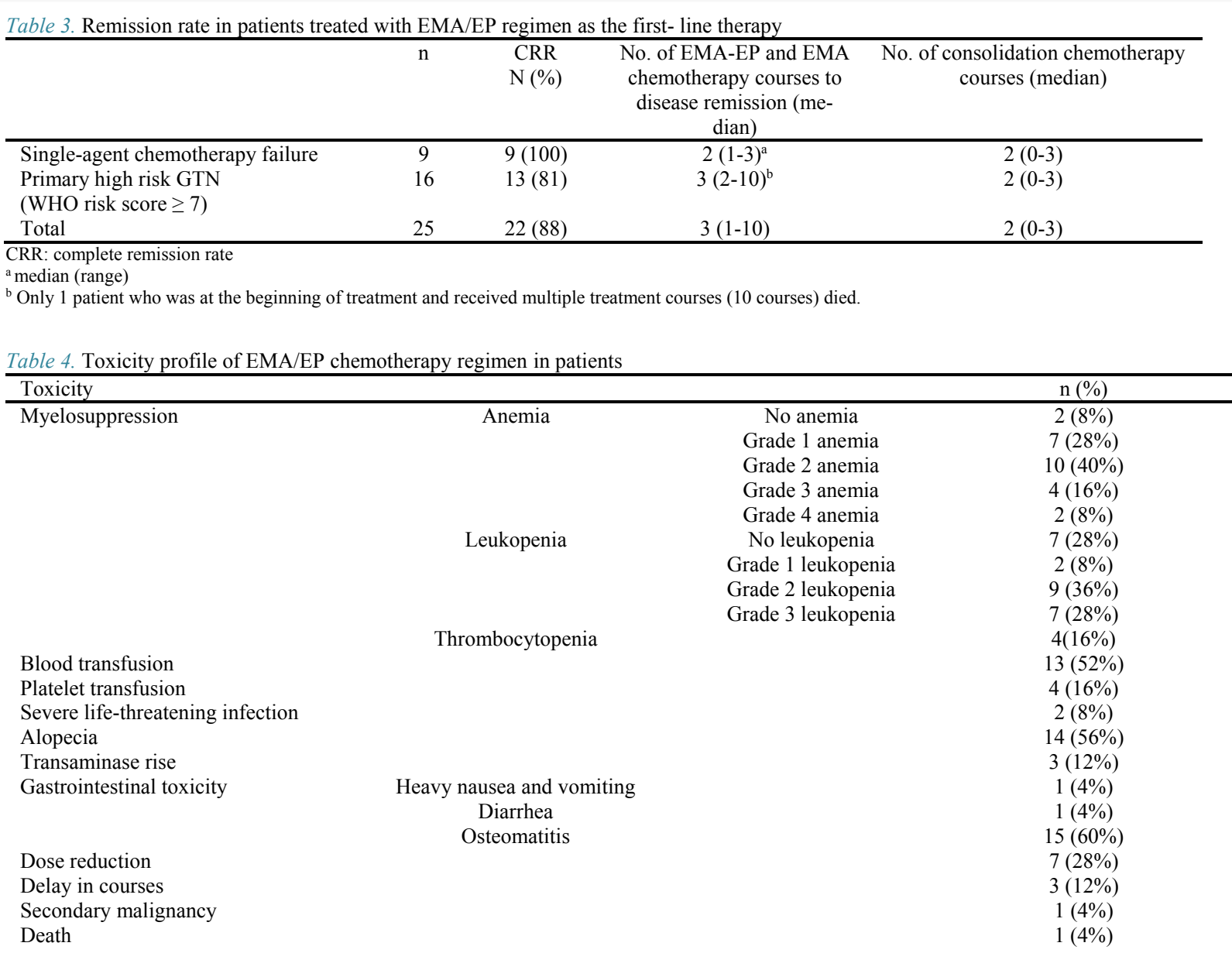

One patient expired due to acute myeloid leukemia (AML), which is the most severe adverse effect of EMA/EP regimen.

\section{Discussion}

This study showed the significant efficacy of EMA/EP chemotherapy regimen as the first- line therapy in the treatment of high-risk GTN. Overall remission rate in GTN patients treated with EMA/EP was $88 \%$, while complete remission rate of $100 \%$ was observed in patients with failure of single agent chemotherapy in primary low-risk score or stage 1 . The most common observed toxic effects of EMA/EP regimen were anemia and leucopenia.

Considering the limitations of EMA/CO, some authors have suggested to use the EMA/EP regimen as the first- line therapy for high-risk GTN. In the first study on this topic by Ghaemmaghami et al. (12) in Iran, 17 patients with highrisk metastatic GTN were treated with EMA/EP regimen as the first- line therapy from 2000 to 2003. In their study, overall remission rate with EMA/EP chemotherapy as the first- line therapy was $88 \%$ and $100 \%$ in patients who completed chemotherapy. This rate was similar to ours because the treatment regimen and protocol was the same. The only difference of that study with ours was in the studied patients. They selected patients with metastatic high-risk GTN, and we selected both metastatic and non-metastatic GTN patients. Moreover, they included patients with WHO risk score of $\geq 6$, while we selected those with a risk score of $\geq 7$. However, the results are comparable in both studies.
In Ghaemmaghami et al. study, similar to ours, anemia and leukopenia were the most common toxicities of EMA/EP regimen, while the rate of these adverse effects in our study was higher than their study and resulted in higher rate of blood transfusion. Although the rate of neutropenia in our study was less than theirs, the need for platelet transfusion in patients was higher in our study than in Ghaemmaghami et al. study. However, the rate of adverse hepatic effects of EMA/EP in both studies was similar. Inclusively, due to equal sample sizes of the high-risk group in Ghaemmaghami et al. and ours, the overall remission rate was the same (12).

In India, Cyriac S et al. (11) evaluated 9 patients with very high-risk GTN who received the EMA/EP regimen as the frontline therapy. The overall survival rate was reported to be $66.7 \%$ and all patients with lung or lung and liver metastasis survived. They concluded that EMA/EP regimen is highly effective in the treatment of patients with high-risk GTN. Remission rate was lower than our study, which may be due to the smaller sample size or other protocol factors.

Hann SN et al. (13) assessed the safety and efficacy of EMA/EP regimen as the first- line therapy or salvage chemotherapy in 18 patients with high-risk GTN. Complete remission rate was $89 \%$, which was similar to our study. In that study, anemia and neutropenia occurred in all patients $(100 \%)$. In their study, 2 patients $(11 \%)$ died due to progressive disease and sepsis and endocarditis. However, mortality rate was $4 \%$ in our study. They concluded that $\mathrm{EMA} / \mathrm{EP}$ with high remission rate and moderate toxicity is 
an excellent regimen for the treatment of high-risk GTN.

EMA/EP regimen has been associated with other toxic effects including myelosuppression and hepatotoxicity, which lead to delaying the treatment and dosage reductions. Administration of granulocyte-colony stimulating factor (G-CSF) to the patients may prevent or reduce myelosuppression and hematologic adverse effects of EMA/EP (2, $14,15)$. In our study, the rate of myelosuppression with EMA/EP regimen was high although all patients received G-CSF. It is a regimen with a high rate of cure that outweighs its toxicity.

The limitation of this study was its retrospective nature, and thus conducting randomized controlled trials is highly suggested for more accurate evaluations.

\section{Conclusion}

According to the results, EMA/EP regimen as the first line- treatment could induce complete remission in $88 \%$ of patients with high-risk GTN. In the previous regimen (EMA/CO), Oncovin (vincristine) was associated with constipation and extravasation and induced skin necrosis. Cyclophosphamide induces hemorrhagic cystitis. One of the late side effects of chemotherapy regimens, including etoposide, is leukemia, which could lead to mortality. Application of EMA/EP may be recommended as the first- line therapy in high-risk GTN patients, especially in patients with failure of single agent chemotherapy. However, necessary steps should be taken to prevent and reduce EMA/EP toxic effects that are tolerable in general.

\section{Conflict of Interests}

The authors declare that they have no competing interests.

\section{References}

1. Aminimoghaddam S, Yarandi F, Nejadsalami F, Taftachi F, Noorbakhsh F, Mahmoudzadeh F. Human chorionic gonadotrophin as an indicator of persistent gestational trophoblastic neoplasia. Med J Islam Repub Iran. 2014;28(1):272-5.

2. Seckl MJ, Sebire NJ, Berkowitz RS. Gestational trophoblastic disease. Lancet. 2010;376(9742):717-29.

3. Altieri A, Franceschi S, Ferlay J, Smith J, La Vecchia C. Epidemiology and aetiology of gestational trophoblastic diseases. Lancet Oncol. 2003;4(11):670-8

4. Aminimoghaddam S, Maghsoudnia A. Unusual Presentation of Invasive Mole: A Case Report. J Reproduct Infertilit. 2017;18(1):2059.

5. Lurain JR. Gestational trophoblastic disease II: classification and management of gestational trophoblastic neoplasia. Am J Obstet Gynecol. 2011;204(1):11-8.

6. Yarandi F, Mousavi A, Abbaslu F, Aminimoghaddam S, Nekuie S, Adabi K, et al. Five-Day Intravascular Methotrexate Versus Biweekly Actinomycin-D in the Treatment of Low-Risk Gestational Trophoblastic Neoplasia: A Clinical Randomized Trial. Int J Gynecol Cancer. 2016;26(5):971-6.

7. Alifrangis C, Agarwal R, Short D, Fisher RA, Sebire NJ, Harvey R, et al. EMA/CO for high-risk gestational trophoblastic neoplasia: good outcomes with induction low-dose etoposide-cisplatin and genetic analysis. J Clin Oncol. 2013;31(2):280-6.

8. Mao Y, Wan X, Lv W, Xie X. Relapsed or refractory gestational trophoblastic neoplasia treated with the etoposide and cisplatin/etoposide, methotrexate, and actinomycin D (EP-EMA) regimen. Int J Gynaecol Obstet. 2007;98(1):44-7.

9. Bower M, Newlands ES, Holden L, Short D, Brock C, Rustin GJ, et al. EMA/CO for high-risk gestational trophoblastic tumors: results from a cohort of 272 patients. J Clin Oncol. 1997;15(7):2636-43.
10. Deng L, Zhang J, Wu T, Lawrie TA. Combination chemotherapy for primary treatment of high-risk gestational trophoblastic tumour. Cochrane Database Syst Rev. 2013;1:CD005196.

11. Cyriac S, Rajendranath R, Sridevi V, Sagar TG. Etoposide, cisplatinetoposide, methotrexate, actinomycin-D as primary treatment for management of very-high-risk gestational trophoblastic neoplasia. Int J Gynaecol Obstet. 2011;115(1):37-9.

12. Ghaemmaghami F, Modares M, Arab M, Behtash N, Moosavi AZ, Khanafshar N, et al. EMA-EP regimen, as firstline multiple agent chemotherapy in high-risk GTT patients (stage II-IV). Int J Gynecol Cancer. 2004;14(2):360-5.

13. Han SN, Amant F, Leunen K, Devi UK, Neven P, Vergote I. EP-EMA regimen (etoposide and cisplatin with etoposide, methotrexate, and dactinomycin) in a series of 18 women with gestational trophoblastic neoplasia. Int J Gynecol Cancer. 2012;22(5):875-80.

14. Lurain JR, Nejad B. Secondary chemotherapy for high-risk gestational trophoblastic neoplasia. Gynecol Oncol. 2005;97(2):618-23.

15. El-Helw LM, Seck1 MJ, Haynes R, Evans LS, Lorigan PC, Long J, et al. High-dose chemotherapy and peripheral blood stem cell support in refractory gestational trophoblastic neoplasia. $\mathrm{Br} \mathrm{J}$ Cancer. 2005;93(6):620-1. 